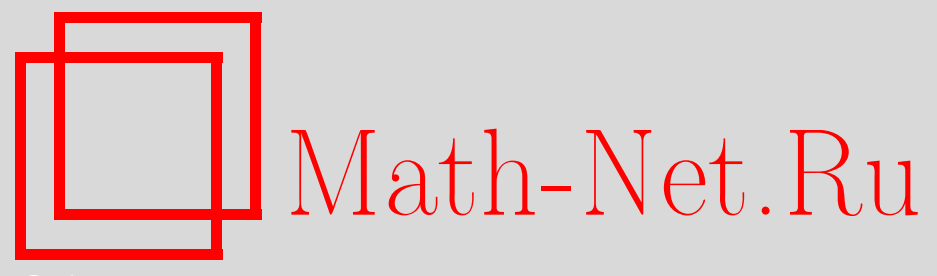

В. П. Елизаров, Условия, необходимые для разрешимости системы линейных уравнений над кольцом, Дискрет. матем., 2004, том 16, выпуск 2, 44-53

DOI: https://doi.org/10.4213/dm151

Использование Общероссийского математического портала Math-Net.Ru подразумевает, что вы прочитали и согласны с пользовательским соглашением http://www . mathnet.ru/rus/agreement

Параметры загрузки:

IP: 54.205 .225 .156

26 апреля 2023 г., 12:59:57 
УдК 512.8

\section{Условия, необходимые для разрешимости системы линейных уравнений над кольцом}

() 2004 г. В. П. Елизаров

Приведены условия, необходимые для разрешимости систем линейных уравнений над ассоциативными кольцами. В некоторых случаях эти условия являются и достаточными для разрешимости систем.

Работа выполнена при поддержке грантом НШ-2358.2003.9 Президента Российской Федерации поддержки ведущих научных школ.

\section{1. Введение}

Везде далее термин кольцо означает ассоциативное кольцо с единицей, отличной от нуля. Если $S-$ подмножество кольца $R$, то через $S_{m, n}$ обозначим совокупность всех $m \times n$ матриц с элементами из $S$, и в частных случаях положим $S(m)=S_{1, m}$ и $S^{(n)}=S_{n, 1}$. Для (правого) идеала $J$ кольца $R$ введем обозначение $J \triangleleft R\left(J \triangleleft_{r} R\right)$.

Рассматривается система линейных уравнений

$$
A X^{\downarrow}=B^{\downarrow}
$$

над кольцом $R$, где $B^{\downarrow}=\left(b_{1}, \ldots, b_{m}\right)^{T} \in R^{(m)}$ и

$$
A=\left(A_{1}^{\downarrow}, \ldots, A_{n}^{\downarrow}\right)=\left(\begin{array}{c}
\vec{A}_{1} \\
\ldots \\
\vec{A}_{m}
\end{array}\right) \in R_{m, n} \backslash\left\{0_{m \times n}\right\} .
$$

Для системы (1) могут выполняться или не выполняться все или некоторые из следующих свойств:

(I) система разрешима;

(II) (1) если $\vec{C} \in R(m)$ и $J \triangleleft_{r} R$, то $(\vec{C} A \in J(n)) \Rightarrow\left(\vec{C} B^{\downarrow} \in J\right)$;

(2) если $\vec{C} \in R(m)$ и $d \in R$, то $(\vec{C} A \in d R(n)) \Rightarrow\left(\vec{C} B^{\downarrow} \in d R\right)$;

(3) если $\vec{C} \in R(m)$, то $(\vec{C} A=\overrightarrow{0}) \Rightarrow\left(\vec{C} B^{\downarrow}=0\right)$;

(IV) (1) если $J_{i} \triangleleft_{r} R, i \in\{1, \ldots, m\}$, то $\left(\vec{A}_{i} \in J_{i}(n)\right) \Rightarrow\left(b_{i} \in J_{i}\right)$;

(2) $d_{i} \in R, i \in\{1, \ldots, m\}$, то $\left(\vec{A}_{i} \in d_{i} R(n)\right) \Rightarrow\left(b_{i} \in d_{i} R\right)$;

(3) если $J \triangleleft_{r} R$, то $\left(A \in J_{m, n}\right) \Rightarrow\left(B^{\downarrow} \in J^{(m)}\right)$; 
(4) если $d \in R$, то $\left(A \in d R_{m, n}\right) \Rightarrow\left(B^{\downarrow} \in d R^{(m)}\right)$.

Пусть $D$ - матрица над коммутативным кольцом $R$. Через $M^{(k)}(D)$ обозначим множество всех ее миноров $k$-го порядка, а через $I^{(k)}(D)$ - идеал кольца $R$, порожденный множеством $M^{(k)}(D)$. Рангом матрицы $D$ называется наибольший из порядков ее ненулевых миноров или нуль, если таких миноров нет (ранг $D$ будем обозначать rank $D$ ).

Для системы уравнений (1) над коммутативным кольцом могут выполняться или не выполняться следующие свойства:

(1) $I^{(k)}(A)=I^{(k)}\left(A, B^{\downarrow}\right), 1 \leqslant k \leqslant \min \{m, n\}$;

(2) $\operatorname{rank} A=\operatorname{rank}\left(A, B^{\downarrow}\right)$.

Соотношения между указанными свойствами демонстрируются диаграммой

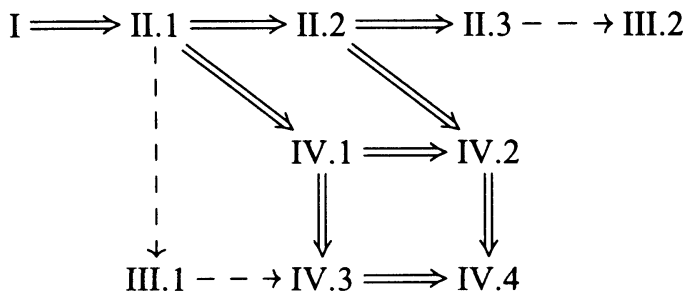

где ни одну из импликаций нельзя заменить на равносильность и нельзя добавить импликацию, не являющуюся композицией уже имеющихся (в некоммутативном случае свойства III.1 и III.2 отсутствуют) [3, 4].

Некоторые свойства из диаграммы (2) являются и достаточными для разрешимости системы (1) над кольцами из того или иного класса колец. Так, свойства II.2 и II.3 были приведены в первом издании книги Ван дер Вардена [13] в качестве необходимых и достаточных условий разрешимости системы соответственно над кольцом $\mathbf{Z}$ целых чисел и над телом. Более обще, выполнение свойства II.3 - критерий разрешимости системы над квазифробениусовым кольцом [2]. Свойства III.1 и III.2 были получены в качестве необходимых и достаточных (в совокупности) для разрешимости системы над кольцом $\mathbf{Z}$ еще в XIX веке (в $[10,11]$ свойство III.1 формулировалось как равенство наибольших общих делителей множеств чисел $M^{(k)}(A)$ и $M^{(k)}\left(A, B^{\downarrow}\right)$, а в качестве критерия разрешимости системы над кольцом целых алгебраических чисел и уже в терминах идеалов в [12]). Свойство II.2 необходимо и достаточно для разрешимости системы (1) над областью главных правых и главных левых идеалов [3].

Свойства группы IV из диаграммы (2) характеризуют разрешимость некоторых уравнений. Выполнение свойства IV.4 или IV.3 - критерий разрешимости соответственно уравнения $a x_{1}=b$ или $a_{1} x_{1}+\ldots+a_{n} x_{n}=b, n>1$, а каждое уравнение системы (1) разрешимо тогда и только тогда, когда выполняется свойство IV.1 (см. [4]).

$\mathrm{B}$ настоящей работе рассматривается еще одна группа свойств (группа V), которые могут выполняться или не выполняться для системы (1), но они необходимы для ее разрешимости, несколько сильнее соответствующих свойств из группы IV и не зависят от свойств групп II и III. Свойства группы V подходят к вопросу о разрешимости системы уравнений (1) со стороны, противоположной той, с которой подходят к этому вопросу свойства группы II: свойство II.3 дает хороший результат, например, если пересечение $M(R)$ ненулевых правых идеалов кольца $R$ отлично от нуля, а свойство V.3 - если $M(R)=\{0\}$. При выполнении свойства V.1 разрешима любая собственная подсистема 
системы уравнений (1). Свойство V.3 обобщает на класс евклидовых областей, не являющихся полями, критерий Минковского-Хассе разрешимости системы уравнений над кольцом $\mathbf{Z}$ (см. стр. 27 в [5]).

В заключение в статье сформулировано несколько проблем.

\section{2. Система уравнений над произвольным кольцом}

Рассмотрим следующие свойства, которые могут выполнятъся или не выполняться для системы уравнений (1) над произвольным кольцом $R$ :

(V) (1) если среди $J_{i} \triangleleft_{r} R, i \in\{1, \ldots, m\}$, существует отличный от нуля идеал, то найдется такой вектор $F^{\downarrow} \in R^{(n)}$, что справедливы включения

$$
\vec{A}_{i} F^{\downarrow}-b_{i} \in J_{i}, \quad i \in\{1, \ldots, m\} ;
$$

(2) $J \triangleleft_{r} R, J \neq\{0\}$ то найдется такой вектор $F^{\downarrow} \in R^{(n)}$, что $A F^{\downarrow}-B^{\downarrow} \in J^{(m)}$;

(3) если $I \triangleleft R, I \neq\{0\}$, то найдется такой вектор $F^{\downarrow} \in R^{(n)}$, что $A F^{\downarrow}-B^{\downarrow} \in I^{(m)}$.

Свойства V.1-V.3 допускают другие формулировки.

Предложение 1. Свойства V.1, V.2 и V.3 для системы уравнений (1) равносильны соответственно свойствам

(V) (1.1) если среди $d_{i} \in R, i \in\{1, \ldots, m\}$, существует отличный от нуля элемент, то найдется такой вектор $G^{\downarrow} \in R^{(n)}$, что

$$
\vec{A}_{i} G^{\downarrow}-b_{i} \in d_{i} R, \quad i \in\{1, \ldots, m\} ;
$$

(2.1) если $d \in R \backslash\{0\}$, то найдется такой вектор $G^{\downarrow} \in R^{(n)}$, что

$$
A G^{\downarrow}-B^{\downarrow} \in d R^{(m)} ;
$$

(3.1) если $I \triangleleft R, I \neq\{0\}$, то над кольчом $\bar{R}=R / I$ разрешима система уравнений

$$
\varphi(A) Y^{\downarrow}=\varphi\left(B^{\downarrow}\right),
$$

где $\varphi: R \rightarrow \bar{R}-$ канонический гомоморфизм.

Доказательство стандартно.

Соотношения между свойствами V.1-V.3 и свойствами из диаграммы (2) в общем случае устанавливает следующая теорема.

Теорема 1. Для системы уравнений (1) над кольчом $R$, в которой $A \neq 0_{m \times n}$, справедливы импликации

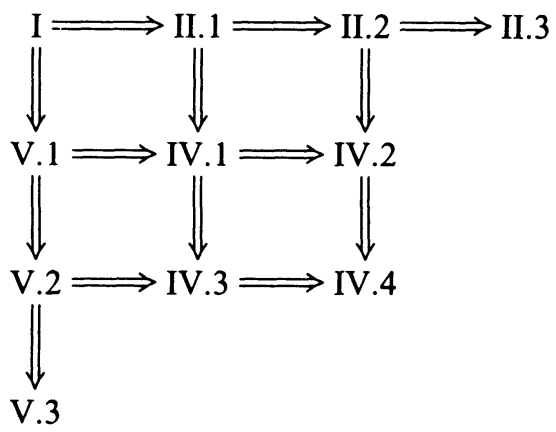


В диаграмме (3) ни одну из импликачий $\Longrightarrow$ нельзя заменить на равносильность и нельзя добавить импликачию, не являючуюся композичией имеючихся.

Доказательство. Импликации I $\Rightarrow$ V.1 $\Rightarrow$ V.2 $\Rightarrow$ V.3 очевидны.

Докажем импликацию V.1 $\Rightarrow$ IV.1. Пусть $J_{i} \triangleleft R, i \in\{1, \ldots, m\}$, и $\vec{A}_{i} \in J_{i}(n)$. Так как $A \neq 0_{m \times n}$, то среди $J_{i}$ существует отличный от нуля идеал. Согласно V.1 найдется такой вектор $F^{\downarrow} \in R^{(n)}$, что $\vec{A}_{i} F^{\downarrow}-b_{i} \in J_{i}, i \in\{1, \ldots, m\}$. Поскольку $\vec{A}_{i} F^{\downarrow} \in J_{i}$, то $b_{i} \in J_{i}$, $i \in\{1, \ldots, m\}$.

Импликация V.2 $\Rightarrow$ IV.3 доказывается аналогично. Учитывая диаграмму (2), получаем, что имеют место все импликации в диаграмме (3).

Для доказательства второй части теоремы построим соответствующие примеры.

1. V.1 не влечет II.3. Для системы уравнений

$$
\left(\begin{array}{l}
1 \\
3
\end{array}\right) x_{1}=\left(\begin{array}{l}
1 \\
1
\end{array}\right)
$$

над кольцом $Z / 4$ выполняется свойство V.1: при $J_{1}=\{0\}, J_{2} \neq\{0\}$ полагаем $F^{\downarrow}=f=1$, а при $J_{1} \neq\{0\}, J_{2}=\{0\}$ полагаем $F^{\downarrow}=f=3$. Свойство II.3 для системы не выполняется, например, при $\vec{C}=(1,1)$.

2. V.2 не влечет IV.2. Для системы уравнений

$$
\left(\begin{array}{l}
2 \\
0
\end{array}\right) x_{1}=\left(\begin{array}{l}
2 \\
2
\end{array}\right)
$$

над кольцом $R=\mathbf{Z} / 4$ выполняется свойство V.2: полагаем $F^{\downarrow}=f=0$. Свойство IV.2 для системы не выполняется, например, при $d_{1}=2, d_{2}=0$.

3. V.3 не влечет IV.4. В кольце $R$ верхнетреугольных $2 \times 2$ матриц над полем $G F(2)$ положим

$$
a=\left(\begin{array}{ll}
0 & 0 \\
0 & 1
\end{array}\right), \quad b=\left(\begin{array}{ll}
0 & 1 \\
0 & 0
\end{array}\right) .
$$

Для уравнения $a x_{1}=b$ выполняется свойство V.3: $F^{\downarrow}=f=0$, так как $b$ принадлежит любому ненулевому идеалу из $R$. Свойство IV.4 для уравнения не выполняется, так как $b \notin a R$.

4. II.3 не влечет IV.4. Для уравнения $2 x_{1}=1$ над кольцом $Z$ выполняется свойство II.3, но свойство IV.4 не выполняется при $d=2$.

5. II.2 не влечет IV.3. Для уравнения $2 x_{1}+x x_{2}=1$ над кольцом $\mathbf{Z}[x]$ выполняется свойство II.2: если $2=d(x) u(x)$ и $x=d(x) v(x)$, то

$$
\begin{gathered}
x d(x) u(x)=2 d(x) v(x), \quad x u(x)=2 v(x), \quad u(x)=2 u_{1}(x), \\
2=d(x) 2 u_{1}(x), \quad 1=d(x) u_{1}(x), \quad d(x)= \pm 1
\end{gathered}
$$

Свойство IV.3 для уравнения не выполняется: если $I$ - идеал, порожденный элементами 2 и $x$, то матрица $(2, x) \in I_{1,2}$, но $1 \notin I$, так как равенство $2 f(x)+x h(x)=1$, где $f(x)=f_{0}+f_{1} x+\ldots+f_{k} x^{k}$, влечет равенство $2 f_{0}=1$, что невозможно.

6. II.1 не влечет V.3. Для системы уравнений

$$
\left(\begin{array}{cc}
2 & x+2 \\
x & 2
\end{array}\right)\left(\begin{array}{l}
x_{1} \\
x_{2}
\end{array}\right)=\left(\begin{array}{c}
2 \\
x+2
\end{array}\right)
$$


над кольцом $R_{1}=\mathrm{Z} / 4[x] /\left(2 x, x^{2}\right)$ выполняется свойство II.1. Действительно, выпишем собственные идеалы кольца $R_{1}$ :

$$
I_{1}=\{0,2\}, \quad I_{2}=\{0, x\}, \quad I_{3}=\{0, x+2\}, \quad I_{4}=\{0,2, x, x+2\} .
$$

Пусть $\vec{C}=\left(c_{1}(x), c_{2}(x)\right)$. Если $\vec{C}\left(\begin{array}{l}2 \\ x\end{array}\right)=0$, то $c_{1}(x) 2=c_{2}(x) x=0$ и $\vec{C}\left(\begin{array}{c}2 \\ x+2\end{array}\right)=0$. Аналогично из $\vec{C}\left(\begin{array}{c}x+2 \\ 2\end{array}\right)=0$ следует, что $\vec{C}\left(\begin{array}{c}2 \\ x+2\end{array}\right)=0$. Предположим, что

$$
\vec{C}\left(\begin{array}{l}
2 \\
x
\end{array}\right)=\vec{C}\left(\begin{array}{c}
x+2 \\
2
\end{array}\right)=2 \in I_{1} .
$$

Тогда

$$
c_{1}(x) x+c_{2}(x)(x+2)=0, \quad c_{1}(x) x=c_{2}(x)(x+2)=0
$$

и $\vec{C}\left(\begin{array}{c}2 \\ x+2\end{array}\right)=0 \in I_{1}$. Таким же образом проверяется справедливость импликации из II. 1 для идеалов $I_{2}$ и $I_{3}$, а для идеалов $I_{4}$ и $R$ она очевидна.

В то же время система не имеет решений: если $\left(a_{1}+b_{1} x, a_{2}+b_{2} x\right)^{T}$ - решение системы, то из первого уравнения получаем, что $a_{2} \in\{0,2\}$, а из второго - что $a_{2} \in\{1,3\}$. Факты неразрешимости системы и справедливости для нее свойства II.1 без доказательства приведены в [3].

Пусть $R_{2}=G F(2)$ и $R=R_{1} \oplus R_{2}$ - внешняя прямая сумма. Для системы уравнений (4), рассматриваемой над кольцом $R$, также справедливо свойство II.1. Если $I=R_{2}-$ идеал кольца $R, \bar{R}=R / I, \varphi: R \rightarrow \bar{R}$ - канонический гомоморфизм, то система

$$
\varphi\left(\left(\begin{array}{cc}
2 & x+2 \\
x & 2
\end{array}\right)\right)\left(\begin{array}{l}
y_{1} \\
y_{2}
\end{array}\right)=\varphi\left(\left(\begin{array}{c}
2 \\
x+2
\end{array}\right)\right)
$$

над кольцом $\bar{R}$ не имеет решений, так как $\bar{R} \cong R_{1}$ и $\left.\varphi\right|_{R_{1}}=\varepsilon$. По предложению 1 для системы (4) над кольцом $R$ не выполняется свойство V.3.

Роль свойства V.1 для системы уравнений (1) показывает следующее утверждение.

Предложение 2. Если для системы уравнений (1) над кольчом $R$ выполняется свойство V.1, то при $m=1$ система разрешима, а при $m>1$ разрешима любая собственная подсистема системы (1).

Доказательство. Первое утверждение следует из теоремы 1, согласно которой из V.1 следует свойство IV.1, равносильное разрешимости любого уравнения системы (см. [4]).

Докажем второе утверждение. Для упрощения обозначений покажем, что разрешима подсистема, состоящая из первых $k<m$ уравнений системы. Выберем правые идеалы кольца $R: J_{i}=\{0\}$ при $i \in\{1, \ldots, k\}$ и $J_{i}=R$ при $i \in\{k+1, \ldots, m\}$. По свойству V.1 найдется такой вектор $F^{\downarrow} \in R^{(n)}$, что справедливы включения $\vec{A}_{i} F^{\downarrow}-b_{i} \in J_{i}$, $i \in\{1, \ldots, m\}$. Ввиду указанного выбора идеалов $J_{i}$ вектор $F^{\downarrow}$ - решение каждого из первых $k$ уравнений системы.

Утверждение, обратное к второму утверждению предложения 2, неверно: в системе уравнений

$$
\left(\begin{array}{l}
1 \\
2
\end{array}\right) x_{1}=\left(\begin{array}{l}
2 \\
6
\end{array}\right)
$$

над кольцом $\mathbf{Z}$ каждое уравнение разрешимо, но свойство V.1 не выполняется, например, при выборе идеалов $J_{1}=\{0\}, J_{2}=3 Z$. 
Систему уравнений

$$
U A V Y^{\downarrow}=U B^{\downarrow}
$$

где $U \in R_{m, m}, V \in R_{n, n}$ - обратимые матрицы, назовем конгруэнтной системе (1) (термин имеется в [7]). Ясно, что конгруэнтные системы уравнений разрешимы или неразрешимы одновременно.

Предложение 3. Если для системы уравнений (1) над кольцом $R$ выполняется свойство V.3, то оно выполняется и для конгруэнтной ей системы уравнений (5).

Доказательство. Пусть $I \triangleleft R, I \neq\{0\}$. По свойству V.3 для системы (1) найдется такой вектор $F^{\downarrow} \in R^{(n)}$, что $A F^{\downarrow}-B^{\downarrow} \in I^{(m)}$. Тогда

$$
U A V \cdot V^{-1} F^{\downarrow}-U B^{\downarrow} \in I^{(m)} .
$$

\section{3. Система уравнений над коммутативным кольцом}

Укажем класс колец, для систем уравнений над которым свойство V.3 достаточно для разрешимости. Коммутативную область целостности, являющуюся кольцом главных идеалов, назовем областью главных идеалов (ГИ-областью). Для кольца $R$ через $M(R)$ обозначим пересечение всех его ненулевых идеалов.

Теорема 2. Пусть $R$ - область главных идеалов и $M(R)=0$. Система уравнений (1) над $R$ разрешима тогда и только тогда, когда для нее выполняется свойство V.3.

Доказательство. Ввиду теоремы 1 покажем только достаточность условия теоремы.

Пусть для системы (1) выполняется свойство V.3. Так как $R-$ ГИ-область, матрица $A$ эквивалентна диагональной матрице, то есть для некоторых обратимых матриц $U \in R_{m, m}$ и $V \in R_{n, n}$ получаем, что $U A V=\operatorname{diag}\left(h_{1}, \ldots, h_{t}\right), t=\min \{m, n\}$ (см. [8] или главу 3 в [1]).

Записав $U A V \cdot V^{-1} X^{\downarrow}=U B^{\downarrow}$ и $U B^{\downarrow}=D^{\downarrow}$, получаем, что система (1) конгруэнтна системе уравнений

$$
\operatorname{diag}\left(h_{1}, \ldots, h_{t}\right) Y^{\downarrow}=D^{\downarrow}
$$

разрешимость которой достаточно установить.

По предложению 3 для системы (6) выполняется свойство V.3. Поскольку $R-$ ГИобласть, свойство V.3 запишем в таком виде: для любого элемента $a \in R \backslash\{0\}$ найдется такой вектор $F^{\downarrow} \in R^{(n)}$, что

$$
\operatorname{diag}\left(h_{1}, \ldots, h_{t}\right) F^{\downarrow}-D^{\downarrow} \in a R^{(m)}
$$

или что

$$
\vec{H}_{k} F^{\downarrow}-d_{k}=a r_{k}, \quad r_{k} \in R, \quad k \in\{1, \ldots, m\}
$$

где $\vec{H}_{k}-k$-я строка матрицы $H=\operatorname{diag}\left(h_{1}, \ldots, h_{t}\right)$.

Если $\vec{H}_{k}=\overrightarrow{0}$, то из (7) получаем, что $d_{k} \in a R$ для любого $a \in R \backslash\{0\}$, то есть что $d_{k} \in M(R)$. По условию тогда $d_{k}=0$, и решением $k$-го уравнения системы (6) в этом случае является любой вектор из $R^{(n)}$. 
Пусть $\vec{H}_{l} \neq \overrightarrow{0}$. Это значит, что $l$-е уравнение системы (6) имеет вид $h_{l} y_{l}=d_{l}, h_{l} \neq 0$. Согласно (7) найдется такой вектор $F^{\downarrow} \in R^{(n)}$, что $\vec{H}_{l} F^{\downarrow}-d_{l}=h_{l} r_{l}, r_{l} \in R$. Значит, $h_{l}\left(f_{l}-r_{l}\right)=d_{l}$, и $l$-ое уравнение разрешимо. Итак, каждое уравнение системы (6) разрешимо, и из вида системы следует, что она разрешима.

Условие, что $M(R)=\{0\}$, в теореме 2 существенно.

Пример 1. Для системы уравнений

$$
\left(\begin{array}{l}
2 \\
3
\end{array}\right) x_{1}=\left(\begin{array}{l}
1 \\
1
\end{array}\right)
$$

над полем $\mathbf{Q}$ рациональных чисел выполняется свойство V.3 и $M(\mathbf{Q})=\mathbf{Q}$. Система не имеет решений.

Примеры колец, удовлетворяющих условиям теоремы 2, дают евклидовы области, не являющиеся полями. Коммутативная область целостности $R$ называется евклидовой областью, если определено отображение $v:(R \backslash\{0\}) \rightarrow(\mathbf{N} \cup\{0\})$, обладающее следующими свойствами:

- если $a, b \in R \backslash\{0\}$, то из $b \mid a$ следует, что $v(b) \leqslant v(a)$;

- для любых $a \in R$ и $b \in R \backslash\{0\}$ найдутся такие элементы $q, r \in R$, что $a=q b+r$, где или $r=0$, или $r \neq 0$ и $v(r)<v(b)$.

Наиболее известные примеры евклидовых областей, кроме полей, - кольца $\mathbf{Z}$ и $P[x]$, где $P$ - поле. Другие примеры можно найти в главе 2 в [7].

Предложение 4. Евклидова область $R$ является ГИ-кольчом. Если $R$ - не поле, то множество $\{v(a) \mid a \in R \backslash\{0\}\}$ неограничено сверху.

Доказательство. Доказательство первого утверждения содержится в главе 6 в [7], а доказательство второго - в главе 2 в [7].

Предложение 5. Класс колеч, удовлетворяющих условиям теоремы 2, содержит класс евклидовых областей, не являющихся полями.

Доказательство. По первому утверждению предложения 4 евклидова область является ГИ-кольцом. Пусть $R$ - евклидова область, не являющаяся полем, и $c \in M(R), c \neq 0$. Так как $R-$ ГИ-кольцо, справедливо включение

$$
c \in \bigcap_{a \in R \backslash\{0\}} a R \text {. }
$$

Значит, $a \mid c$ для любого элемента $a \in R \backslash\{0\}$. Тогда $v(a) \leqslant v(c)$. Это противоречит второму утверждению предложения 4. Следовательно, $M(R)=\{0\}$.

Пример 1 показывает, что требование не быть полем существенно в предложении 5.

Теорема 2 и предложение 5 показывают, для систем уравнений над какими кольцами можно обобщить принцип Минковского-Хассе: система уравнений (1) над кольцом $\mathbf{Z}$ разрешима тогда и только тогда, когда для любого $k \in \mathbf{N} \backslash\{1\}$ разрешима система сравнений $A X^{\downarrow} \equiv B^{\downarrow}(\bmod k)$ (см. стр. 27 в [5]). Принцип Минковского-Хассе - это свойство V.3.1, которое по предложению 1 равносильно свойству V.3.

Установим теперь соотношения между свойствами групп II, III, IV и V в случае коммутативного кольца. 
Теорема 3. Для системы уравнений (1) над коммутативным кольчом $R$, в которой $A \neq$ $0_{m \times n}$, справедливы импликачии

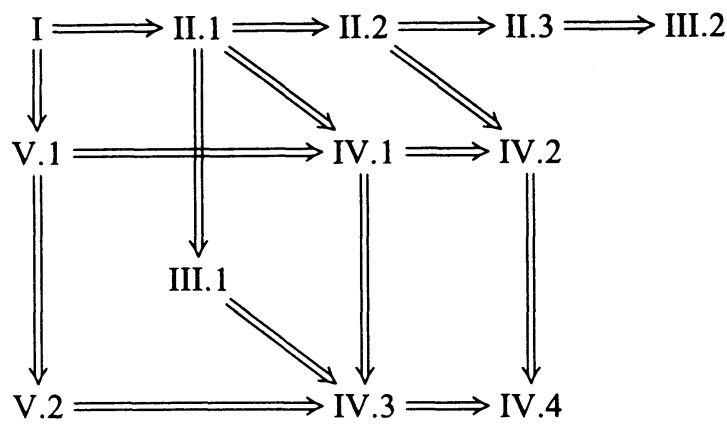

В диаграмме (8) ни одну из импликаций $\Longrightarrow$ нельзя заменить на равносильность $и$ нельзя добавить импликачию, не являючуюся композичией имеющихся.

Доказательство. Импликации II.1 $\Rightarrow$ III.1 $\Rightarrow$ IV.3 и II.3 $\Rightarrow$ III.2 доказаны в [3] и [4]. Вместе с диаграммой (3) этим показано, что все импликации в диаграмме (8) имеют место.

Для доказательства второй части теоремы укажем соответствующие примеры.

1. V.1 не влечет III.2. Для системы уравнений

$$
\left(\begin{array}{l}
e \\
e
\end{array}\right) x_{1}=\left(\begin{array}{l}
0 \\
e
\end{array}\right)
$$

над полем $G F(2)$ выполняется свойство V.1: если $J_{1}=\{0\}, J_{2}=G F(2)$, то $f=0$, а если $J_{1}=G F(2), J_{2}=\{0\}$, то $f=e$. Однако свойство III.2 не выполняется.

2. III.1 не влечет V.2. Для системы уравнений

$$
\left(\begin{array}{l}
1 \\
2
\end{array}\right) x_{1}=\left(\begin{array}{l}
3 \\
3
\end{array}\right)
$$

над кольцом $\mathbf{Z}$ выполняется свойство III.1. Но свойство V.3 не выполняется: при $\boldsymbol{J}=5 \mathbf{Z}$ не могут выполняться включения $f-3 \in 5 \mathrm{Z}$ и $2 f-3 \in 5 \mathrm{Z}$.

3. V.1 не влечет III.1. Для системы уравнений

$$
\left(\begin{array}{ll}
3 & 0 \\
3 & 0
\end{array}\right)\left(\begin{array}{l}
x_{1} \\
x_{2}
\end{array}\right)=\left(\begin{array}{l}
2 \\
0
\end{array}\right)
$$

над кольцом $\mathbf{Z} / 4$ выполняется свойство V.1: $3 f-2 \in(2), 3 f=0$ при $f=0$ и $3 f-2=0$, $3 f \in(2)$ при $f=2$. Свойство III.1 для системы не выполняется, так как

$$
I^{(2)}\left(\left(\begin{array}{ll}
3 & 0 \\
3 & 0
\end{array}\right)\right)=\{0\}, \quad I^{(2)}\left(\left(\begin{array}{lll}
3 & 0 & 2 \\
3 & 0 & 0
\end{array}\right)\right)=\{0,2\} .
$$

Диаграммы (2) и (3), в которых ни одну из импликаций $\Longrightarrow$ нельзя заменить на равносильность и нельзя добавить импликацию, не являющуюся комбинацией имеющихся, доказывают вторую часть теоремы. 
Произвольное кольцо $R$ называется квазифробениусовым ( $Q F$-кольцом), если оно артиново справа и слева и для любого его правого идеала $J$ и левого идеала $I$ справедливы равенства

$$
\operatorname{Ann}_{r} \operatorname{Ann}_{l} J=J, \quad \operatorname{Ann}_{l} \operatorname{Ann}_{r} I=I .
$$

Примерами $Q F$-колец служат тела и кольца вычетов $\mathbf{Z} / n$ и $G F(q)[x] / f(x)$. Система уравнений над $Q F$-кольцом разрешима тогда и только тогда, когда для нее выполняется свойство II.3 (см. [2]).

Поэтому теорема 2 и тот факт, что для локального $Q F$-кольца $R$ идеал $M(R)$ отличен от нуля (см., например, [6]), показывают, что группы свойств II и V не только независимы (теоремы 1 и 3), но и подходят к вопросу о разрешимости системы уравнений (1) с разных сторон.

\section{4. Проблемы}

(1) Указать какой-либо класс колец, для систем уравнений над которыми критерием разрешимости является выполнение свойства I.1 или V.1.

(2) Описать класс всех колец (всех коммутативных колец), для систем уравнений над которыми критерием разрешимости является выполнение одного из свойств группы II или группы V.

По-видимому, такое описание является трудной задачей. Однако в случае систем уравнений, состоящих из бесконечного множества уравнений от бесконечного множества неизвестных таких, что в каждое уравнение системы входит только конечное число неизвестных, класс колец, для систем уравнений над которыми критерием разрешимости является выполнение свойства II.3 - это в точности класс классически полупростых колец [9]. В [3] показано, что класс II.1- (II.2- или II.3-) разрешимых колец замкнут относительно конечных прямых сумм и перехода к кольцам матриц, а классы II.1-разрешимых или II.3-разрешимых колец замкнуты и относительно гомоморфных образов. Под классом II.1-разрешимых колец понимаем класс колец, для которых критерием разрешимости системы (1) является выполнение свойства II.1 (аналогично определяются II.2-разрешимые и II.3-разрешимые кольца).

\section{Список литературы}

1. Джекобсон Н., Теория колеч. ГИиЛ, Москва, 1947.

2. Елизаров В. П., Системы линейных уравнений над квазифробениусовыми кольцами. Фундам. и прикл. матем. (1995) 1, №2, 535-539.

3. Елизаров В. П., Условия совместности систем линейных уравнений над кольцами. Фундам. $u$ прикл. матем. (2000) 6, №3, 777-788.

4. Елизаров В. П., Системы линейных уравнений над модулями. Фундам. и прикл. матем. (2003) 9 (2003), №4, в печати.

5. Манин Ю. И., Панчишкин А. А., Введение в теорию чисел. Итоги науки и техники. Современные проблемы математики. Фундаментальные направления. (1989) 49, 5-348.

6. Нечаев А. А., Критерии полноты систем функций на конечном кольце и квазифробениусовы кольца. Сиб. матем. журиал (1982) 23, №3, 175-182. 
7. Родосский К. А., Алгоритм Евклида. Наука, Москва, 1988.

8. Asano K., Über verallgemeinerte Abelsche Gruppen mit hypercomplexem Operatorenring und ihre Anwendungen. Japan J. Math. (1939) 15, №4, 231-253.

9. Kertesz A., The general theory of linear equation systems over semisimple rings. Publ. Math. Debrecen (1955) 4, №1-2, 79-86.

10. Smith H. J. S., On systems of linear indeterminate equations and congruences. Phil. Trans. Royal Soc. London (1861) A151, 293-326.

11. Smith H. J. S., On the arithmethical invariants of rectangular matrix of which the constituents are integral numbers. Proc. London Math. Soc. (1873) 4, 236-249.

12. Steinitz E., Rechteckige Systeme und Moduln in algebraischen Zahlkorpern. I. Math. Ann. (1912) 71, №3, 328-354.

13. Van der Waerden B. L., Moderne Algebra. II. Springer, Berlin, 1931.

Статья поступила 22.04.2003. 